PROCEEDINGS OF THE

AMERICAN MATHEMATICAL SOCIETY

Volume 129, Number 7 , Pages $2111-2117$

S 0002-9939(01)05855-5

Article electronically published on February 15, 2001

\title{
LATTICE PROPERTIES OF SUBSPACE FAMILIES IN AN INNER PRODUCT SPACE
}

\author{
PAVEL PTÁK AND HANS WEBER
}

(Communicated by David R. Larson)

\begin{abstract}
Let $S$ be a separable inner product space over the field of real numbers. Let $E(S)$ (resp., $C(S)$ ) denote the orthomodular poset of all splitting subspaces (resp., complete-cocomplete subspaces) of $S$. We ask whether $E(S)$ (resp., $C(S)$ ) can be a lattice without $S$ being complete (i.e. without $S$ being Hilbert). This question is relevant to the recent study of the algebraic properties of splitting subspaces and to the search for "nonstandard" orthomodular spaces as motivated by quantum theories. We first exhibit such a space $S$ that $E(S)$ is not a lattice and $C(S)$ is a (modular) lattice. We then go on showing that the orthomodular poset $E(S)$ may not be a lattice even if $E(S)=C(S)$. Finally, we construct a noncomplete space $S$ such that $E(S)=C(S)$ with $E(S)$ being a (modular) lattice. (Thus, the lattice properties of $E(S)$ (resp. $C(S)$ ) do not seem to have an explicit relation to the completeness of $S$ though the Ammemia-Araki theorem may suggest the opposite.) As a by-product of our construction we find that there is a noncomplete $S$ such that all states on $E(S)$ are restrictions of the states on $E(\bar{S})$ for $\bar{S}$ being the completion of $S$ (this provides a solution to a recently formulated problem).
\end{abstract}

\section{Preliminaries}

This paper continues to study algebraic properties of splitting subspaces (see [1, [2], 3], 7], 8, [14, etc.) and contributes to the investigation of "nonstandard" orthomodular spaces (see [4, 9], [11, 13, 16], etc.). Let us first review basic notions, as we shall use them in the sequel.

Let $S$ be an inner product space over the reals and let $(\cdot, \cdot)$ be the inner product on $S$. Let us set, for each subset $A$ of $S, A^{\perp}=\{b \in S \mid(a, b)=0$ for any $a \in A\}$. Let us further denote by $E(S)$ the set of all subspaces $A$ of $S$ such that $A+A^{\perp}=S$. Using the symbol $\oplus$ to denote the sum of orthogonal spaces, we have $E(S)=\{A \subset$ $\left.S \mid A \oplus A^{\perp}=S\right\}$.

Let us endow $E(S)$ with the partial ordering given by inclusion $\subset$ and with the above defined orthocomplementation $\perp$. Then $E(S)$ can be viewed as an orthocomplemented poset and will be looked at this way in the sequel.

Received by the editors September 30, 1998 and, in revised form, June 2, 1999

2000 Mathematics Subject Classification. Primary 03G12, 46C05, 81P10.

Key words and phrases. Inner product (= prehilbert) space, splitting subspace, orthomodular poset, lattice.

The authors acknowledge the support of grant GAČR 201/98/1153 of the Czech Grant Agency and Progetto di ricerca di interesse nazionale Analisi Reale (Italy).

(C)2001 American Mathematical Society 
Let $C(S)$ be the collection of all complete and all cocomplete subspaces of $S$ (by a cocomplete subspace of $S$ we mean a subspace $B$ such that $B=A^{\perp}$ in $S$ for a complete subspace $A$ ). As we show in the following Proposition 1.1, the set $C(S)$ is a subset of $E(S)$. If we endow $C(S)$ with the partial ordering and orthocomplementation inherited from $E(S)$, then we obtain another orthocomplemented poset. Both sets $E(S)$ and $C(S)$ in fact form an orthomodular poset. Let us show this, together with some other useful properties, in Proposition 1.1 Though most of the properties are known (see [14] and 2]), we collect them for the convenience of the reader. We also want to arrange them so that an explicit reference is possible.

Proposition 1.1. Let $S$ be an inner product space and let $E(S)$ and $C(S)$ be orthocomplemented posets defined above. Then:

(i) if $A$ is complete or cocomplete, then $A \in E(S)$,

(ii) if $A, B \in E(S)$ and $A \subset B^{\perp}$, then $A \oplus B \in E(S), A \oplus B=A \vee B$ in $E(S)$, $(A \oplus B)^{\perp}=A^{\perp} \cap B^{\perp}$, and $A^{\perp} \cap B^{\perp} \in E(S)$; in particular, $A^{\perp} \cap B^{\perp}=A^{\perp} \wedge B^{\perp}$ in $E(S)$,

(iii) if $v \in S$ and $A \in E(S)$, then $A+[v] \in E(S)$ (the symbol $[v]$ denotes the span of $v$ in $S)$,

(iv) $E(S)$ is an orthomodular poset (for the definition of orthomodular poset, see e.g. [15]),

(v) $C(S)$ is an orthomodular poset (in fact, $C(S)$ is a suborthomodular poset of $E(S))$

(vi) for any $A, B \in E(S)$, if the infimum $A \wedge B$ exists in $E(S)$, then $A \wedge B=A \cap B$.

Proof. (i) Suppose that $A$ is a complete subspace of $S$ and suppose further that $H$ is the completion of $S$. Obviously, $A$ is a closed subspace of $H$. Let $A^{\prime}$ be the orthocomplement of $A$ in $H$. Then $H=A \oplus A^{\prime}$ and, moreover, $A^{\perp}=A^{\prime} \cap S$. Since $A \subset S$, we obtain $S=A \oplus\left(A^{\prime} \cap S\right)$. As a consequence, both $A$ and $A^{\perp}$ belong to $E(S)$.

(ii) Let us show that $(A \oplus B) \oplus C=S$, where $C=A^{\perp} \cap B^{\perp}$. Observing that $A^{\perp} \cap B^{\perp}=(A+B)^{\perp}$, let us take $d \in S$. Then $d=a_{1}+\tilde{a}_{1}$, where $a_{1} \in A$ and $\tilde{a}_{1} \in A^{\perp}$, and, also, $d=b_{1}+\tilde{b}_{1}$, where $b_{1} \in B$ and $\tilde{b}_{1} \in B^{\perp}$. Thus, $d=\left(a_{1}+b_{1}\right)+c$, where $c=d-a_{1}-b_{1}$. It follows that $c=\tilde{a}_{1}-b_{1}$. Moreover, $\tilde{a}_{1} \in A^{\perp}$ and $b_{1} \in B \subset A^{\perp}$. As a consequence, $c \in A^{\perp}$. Analogously, $c \in B^{\perp}$. Thus, $c \in A^{\perp} \cap B^{\perp}$. We have shown that $(A \oplus B) \oplus\left(A^{\perp} \cap B^{\perp}\right)=S$. Hence, both $A \oplus B$ and $A^{\perp} \cap B^{\perp}$ belong to $E(S)$. Thus, $A \vee B=A \oplus B$ and $A^{\perp} \wedge B^{\perp}=A^{\perp} \cap B^{\perp}$ in $E(S)$.

(iii) Write $v=v_{1}+v_{2}$, where $v_{1} \in A$ and $v_{2} \in A^{\perp}$. Then $A+[v]=A \oplus\left(v_{2}\right)$, but the latter space obviously belongs to $E(S)$ (Proposition 1.1 (ii)).

(iv) We must verify the orthomodular law in $E(S)$. Thus, we must prove that for each couple $A, B \in E(S)$ with $A \subset B$, the infimum $B \wedge A^{\perp}$ and the supremum $A \vee\left(B \wedge A^{\perp}\right)$ exist and, moreover, we have the equality $B=A \vee\left(B \wedge A^{\perp}\right)$. Since $A \subset B$, we have $B=B \cap\left(A \oplus A^{\perp}\right)=A \oplus\left(B \cap A^{\perp}\right)$. Applying Proposition 1.1(ii) to the couple $A, B^{\perp}$, we obtain that the infimum $A^{\perp} \wedge B$ exists in $E(S)$ and, moreover, $A^{\perp} \wedge B=A^{\perp} \cap B$. As the spaces $A$ and $A^{\perp} \wedge B$ are orthogonal, the supremum $A \vee\left(A^{\perp} \wedge B\right)$ exists and $A \vee\left(A^{\perp} \wedge B\right)=A \oplus\left(A^{\perp} \wedge B\right)$. Hence, $B=A \vee\left(B \wedge A^{\perp}\right)$.

(v) Let $A, B \in C(S)$ and let $A \subset B^{\perp}$. By Proposition 1.1 (i), (ii), the supremum $A \vee B$ exists in $E(S)$ and $A \vee B=A \oplus B$. It only remains to be shown that $A \oplus B \in C(S)$, but this is easy (one only observes that if $S$ is not complete, then both $A$ and $B$ cannot be complete). 
(vi) Write $C=A \wedge B$. Then $C \subset A \cap B$. If there is a vector $v \in S$ which belongs to $(A \cap B) \backslash C$, then $C+[v] \in E(S)$ and therefore $C+[v] \subset A$ and $C+[v] \subset B$. This means that $C \neq A \wedge B$ which is a contradiction.

\section{Results}

Let us now formulate and prove the results we announced in the abstract.

Theorem 2.1. Let $S$ be an inner product space and let $S$ possess a countable linear basis. Then $E(S)$ is not a lattice. Moreover, $C(S)$ consists of all finite dimensional and cofinite dimensional subspaces of $S$ and therefore $C(S)$ is a modular lattice.

Proof. The proof uses the technique of Theorem 4 in [11]. Let us first prove that $E(S)$ is not a lattice. Let us divide the proof into a few propositions.

Proposition 2.1.1. Let $S_{1}, S_{2}$ be inner product spaces and let both $S_{1}, S_{2}$ have a countable linear basis. Then $S_{1}$ and $S_{2}$ are isomorphic as inner product spaces. Thus, $E\left(S_{1}\right)$ and $E\left(S_{2}\right)$ are isomorphic as orthomodular posets.

Proof. Let $\left\{a_{i} \mid i \in N\right\}$ (resp. $\left\{b_{i} \mid i \in N\right\}$ ) be countable linear bases of $S_{1}$ (resp., $S_{2}$ ). Using the Gram-Schmidt orthogonalization process we can construct the corresponding orthonormal bases $\left\{e_{i} \mid i \in N\right\}$ (resp. $\left\{f_{i} \mid i \in N\right\}$ ) of $S_{1}$ (resp. $S_{2}$ ). The coordinate assignment then yields the required inner product isomorphism.

Proposition 2.2.2. Let $c_{00}$ denote the space of all sequences of reals whose coordinates are all 0 up to a finite set. Take the vector $v=\left(1, \frac{1}{2}, \frac{1}{3}, \ldots, \frac{1}{n}, \ldots\right) \in l^{2}(N)$ and put $S=c_{00} \oplus[v]$ (S understood as an $l^{2}$ inner product subspace). Then $E(S)$ is not a lattice.

Proof. Let $f_{n}$ denote the "Cronecker delta" vector in $S$, i.e., let $\left(f_{n}\right)_{m}=\delta_{n}^{m}$, where $\delta_{n}^{m}=1$ if $m=n$ and $\delta_{n}^{m}=0$ otherwise. Let $F_{1}=\operatorname{span}\left\{f_{n} \mid n \in N, n\right.$ is even $\}$ and $F_{2}=\operatorname{span}\left\{f_{n} \mid n \in N, n\right.$ is odd $\}$. Then we easily see that $F_{1} \oplus F_{2}=c_{00}$. The rest will be established in a series of statements.

Statement 1. In $S, F_{1}^{\perp}=F_{2}$, and $F_{2}^{\perp}=F_{1}$.

Indeed, we first see that $F_{2} \subset F_{1}^{\perp}$. In order to check the inclusion $F_{1}^{\perp} \subset F_{2}$, take $b \in c_{00}$ and $\alpha \in R$ such that $a=b+\alpha \cdot v \in F_{1}^{\perp}$. Then $\alpha=0$ (otherwise the vector $a$ cannot have "almost all" coordinates equal to 0 ). Thus, $a \in F_{2}$ and therefore $F_{1}^{\perp} \subset F_{2}$.

Statement 2. $F_{2}+[v] \notin E(S)$.

Indeed, suppose that it is not the case and look for a contradiction. If $F_{2}+$ $[v] \in E(S)$, then $\left(F_{2}+[v]\right) \oplus\left(F_{2}+[v]\right)^{\perp}=S=\left(F_{2}+[v]\right)+F_{1}$. Obviously, $\left(F_{2}+[v]\right)^{\perp} \subset F_{2}^{\perp}=F_{1}$. This implies that $F_{1}=\left(F_{2}+[v]\right)^{\perp}$ and hence $v \in F_{1}^{\perp}$. This is absurd.

Statement 3. Let

$$
\begin{aligned}
& A=\operatorname{span}\left\{2 n \cdot f_{2 n}-(2 n+2) \cdot f_{2 n+2} \mid n \in N, n \text { is odd }\right\}, \\
& B=\operatorname{span}\left\{(2 n+2) \cdot f_{2 n}+2 n \cdot f_{2 n+2} \mid n \in N, n \text { is odd }\right\} .
\end{aligned}
$$

Then $v \in A^{\perp}, A \oplus B=F_{1}$ and $\left(F_{2}+[v]+B\right) \oplus A=S$. Thus, $\left(F_{2}+[v]+B\right) \in E(S)$.

Indeed, it is a matter of straightforward verification that $A$ and $B$ have been constructed in such a manner that they satisfy the last three properties. 
Statement 4. Let us set

$$
\begin{aligned}
& C=\operatorname{span}\left\{(2 n+2) \cdot f_{2 n+2}-(2 n+4) \cdot f_{2 n+4} \mid n \in N, n \text { is odd }\right\}, \\
& D=\operatorname{span}\left\{f_{2}\right\}+\operatorname{span}\left\{(2 n+4) \cdot f_{2 n+2}+(2 n+2) \cdot f_{2 n+4} \mid n \in N, n \text { is odd }\right\} .
\end{aligned}
$$

Then $v \in C^{\perp}, C \oplus D=F_{1}, C \oplus\left(F_{2}+[v]+D\right)=S$ (and, hence, $F_{2}+[v]+D \in E(S)$ ), and $B \cap D=\{o\}$ (the symbol $o$ denotes the zero vector).

Indeed, one can easily check that all properties but the last one can be verified by a straightforward computation. Let us verify the last property. We want to show that $B \cap D=\{o\}$. Let us suppose that $x \in B \cap D$. Then

$$
\begin{aligned}
x & =\sum_{\substack{n=1, n \text { is odd }}}^{p} \alpha_{n} \cdot\left((2 n+2) \cdot f_{2 n}+2 n \cdot f_{2 n+2}\right) \\
& =\beta_{0} \cdot f_{2}+\sum_{\substack{n=1, n \text { is odd }}}^{q} \beta_{n} \cdot\left((2 n+4) \cdot f_{2 n+2}+(2 n+2) \cdot f_{2 n+4}\right) .
\end{aligned}
$$

If $x \neq o$, then we may assume that $\alpha_{p} \neq 0$ and $\alpha_{q} \neq 0$. Then $2 p+2$ (resp. $2 q+4$ ) is the greatest index for which the coefficient of $f_{i}$ in the first sum (resp. the second sum) is distinct from 0 . But then $2 p+2=2 q+4$ which is impossible in view $p$ and $q$ being odd. It follows that $B \cap D=\{o\}$.

We are in the position to return to Theorem 2.1 and complete the proof. Let $K=F_{2}+[v]+B$ and $L=F_{2}+[v]+D$. We have already proved that both $K$ and $L$ belong to $E(S)$. Let us show that $K \cap L=F_{2}+[v]$. Suppose that $z \in K \cap L$. Then $z=x+b=y+d$, where $x, y \in F_{2}+[v]$ and $b \in B, d \in D$. Then $b-d=y-x \in F_{2}+[v]$. From the definition of $B, D$ and $F_{2}$ we immediately see that $b-d=o$. Thus, $b=d$ and since $B \cap D=\{o\}$, we see that $b=d=o$. This means that $z=x \in F_{2}+[v]$. But $F_{2}+[v]$ does not belong to $E(S)$. In view of Proposition 1.1 (vi), the infimum $K \wedge L$ does not exist in $E(S)$. Thus, $E(S)$ is not a lattice.

To complete the proof of Theorem 2.1 it remains to be shown that $C(S)$ consists of all finite dimensional and cofinite dimensional subspaces of $S$. This is obvious because each Hilbert space has either a finite or uncountable linear dimension (see e.g. [5]).

In the rest of the paper we will examine dense hyperplanes in a Hilbert space. The reason is obvious from the following proposition.

Proposition 2.2.3. Let $H$ be a Hilbert space and let $S$ be a dense linear hyperplane in $H$ (i.e., let $S$ be a dense subspace of $H$ with $\operatorname{dim} H / S=1$ ). Then $E(S)=C(S)$.

Proof. Let $A \in E(S)$ and $B=A^{\perp}$. Then $S=A \oplus B$. Passing to the completion and observing that $\overline{A \oplus B}=\bar{A} \oplus \bar{B}$, we see that $H=\bar{S}=\bar{A} \oplus \bar{B}$. By a straightforward linear algebra computation, we obtain $\operatorname{dim} \bar{A} / A+\operatorname{dim} \bar{B} / B=\operatorname{dim} H / S=1$. Therefore either $\operatorname{dim} \bar{A} / A=1$, in which case $\bar{B}=B$ (i.e., $B$ is complete) or $\operatorname{dim} \bar{B} / B-1$, in which case $\bar{A}=A$ (i.e., $A$ is complete). It follows that $E(S)=C(S)$.

Remark 2.2.4. It is interesting to observe that if $S$ is a dense linear hyperplane in $H$, then the orthomodular poset $E(S)$ possesses a two-valued state $s: E(S) \rightarrow\{0,1\}$. Indeed, we then have $E(S)=C(S)$ and $C(S)$ always possesses such a state: Since each $A \in C(S)$ is either complete or cocomplete, it suffices to let $s(A)=1$ if, and only if, $A$ is cocomplete. The presence of two-valued states on $E(S)$, for 
$S$ incomplete, sheds light on problems studied in [2], Chapter 4 (for an explicit consequence, see the comments at the end of this paper).

Prior to the next result, let us recall a known property of infinite dimensional Hilbert spaces.

Proposition 2.2.5. Let $H$ be an infinite dimensional Hilbert space. Then there are closed subspaces $A_{0}$ and $B_{0}$ of $H$ such that $A_{0}+B_{0}$ is not closed in $H$ and, moreover, $\operatorname{dim}\left(A_{0}^{\perp} \cap B_{0}^{\perp}\right)=\infty$.

Proof. Let $\left\{b_{1}, b_{2}, \ldots, b_{n}, \ldots\right\}$ be an orthonormal sequence of $H$. Let $A_{0}=$ $\overline{\operatorname{span}}\left\{b_{3 n} \mid n \in N\right\}$ and $B_{0}=\overline{\operatorname{span}}\left\{b_{3 n}+\frac{1}{n} b_{3 n+1} \mid n \in N\right\}$. Then the vector $v=$ $\sum_{n \in N} \frac{1}{n} b_{3 n+1}$ does belong to $\overline{A_{0}+B_{0}}$ but $v$ does not belong to $A_{0}+B_{0}$. Moreover, $A_{0}^{\perp} \cap B_{0}^{\perp} \supset \operatorname{span}\left\{b_{3 n+2} \mid n \in N\right\}$. The proof is complete.

Our next result shows that $E(S)$ may not be a lattice even if $E(S)=C(S)$. Prior to its formulation, let us collect some properties of hyperplanes.

Proposition 2.2.6. Let $S$ be a dense hyperplane in a Hilbert space $H$. Then the following statements hold true:

(i) If $A$ is a closed subspace of $S$ and $\bar{A}$ is the closure of $A$ in $H$, then there is a vector $x \in H$ such that $\bar{A}=A+[x]$.

(ii) If $A, B \in C(S)$ and $A \cap B$ is not complete, then $\bar{A} \cap \bar{B}=\overline{A \cap B}$.

Proof. (i) Since $\bar{A} \cap S=A$, we obtain $\operatorname{dim} \bar{A} / A \leq \operatorname{dim} H / S=1$. This proves the first statement.

(ii) Let $x \in \overline{A \cap B}-(A \cap B)$. Then $x \notin S$ and therefore $x \in \bar{A} \backslash A$ and $x \in \bar{B} \backslash B$. This yields (Proposition 2.2.6 (i)) that $\overline{A \cap B}=(A \cap B)+[x], \bar{A}=A+[x]$ and $\bar{B}=B+[x]$. Let us prove that $\bar{A} \cap \bar{B}=\overline{A \cap B}$. Suppose $y \in \bar{A} \cap \bar{B}$. We can write $y=a+\alpha x=b+\beta x$ for some $\alpha, \beta \in R$ and $a \in A, b \in B$. This implies that $(\alpha-\beta) x=b-a \in S$ and since $x \notin S$, we obtain $\alpha-\beta=0$. Thus, $\alpha=\beta$ and $a=b$. As a consequence, $y=a+\alpha x \in(A \cap B)+x$ and this implies that $y \in \overline{A \cap B}$.

Theorem 2.2.7. Let $H$ be a Hilbert space. Then there is a dense hyperplane, $S$, in $H$ such that $E(S)$ is not a lattice.

Proof. Let $A_{0}, B_{0}$ be closed subspaces of $H$ with the property of Proposition 2.2.5 Choose a vector $x_{0} \in H$ so that $x_{0} \in \overline{A_{0}+B_{0}}-\left(A_{0}+B_{0}\right)$. Write $A_{1}=A_{0}^{\prime}$ and $B_{1}=B_{0}^{\prime}$ (again $A_{0}^{\prime}$ and $B_{0}^{\prime}$ denote the complements of $A_{0}$ and $B_{0}$ in $H$ ). By our construction, $\operatorname{dim}\left(A_{1} \cap B_{1}\right)=\infty$ and therefore there is a dense hyperplane, $S_{1}$, in $A_{1} \cap B_{1}$. It follows that there exists $x_{1} \in H$ such that $S_{1}+\left[x_{1}\right]=A_{1} \cap B_{1}$. We can then write $H=\left(A_{1} \cap B_{1}\right) \oplus \overline{A_{0}+B_{0}}=\left(S_{1}+\left[x_{1}\right]\right) \oplus\left(\left[x_{0}\right]+A_{0}+B_{0}+C\right)$ for a subspace $C$ of $H$. Thus, $x_{0} \notin S_{1}+A_{0}+B_{0}+\left[x_{0}+x_{1}\right]$ because if it is not the case, we have the equation $x_{0}=s_{1}+a_{0}+b_{0}+\alpha\left(x_{0}+x_{1}\right)$, where $s_{1} \in S_{1}, a_{0} \in A_{0}$, $b_{0} \in B_{0}$. This gives us the equation

$$
s_{1}+\alpha x_{1}=(1-\alpha) x_{0}-a_{0}-b_{0} .
$$

Since $s_{1}+\alpha x_{1} \in A_{1} \cap B_{1}$ and $(1-\alpha) x_{0}-a_{0}-b_{0} \in \overline{A_{0}+B_{0}}=\left(A_{1} \cap B_{1}\right)^{\perp}$, we obtain $s_{1}+\alpha x_{1}=0$ which is impossible.

Let $S$ be a dense hyperplane in $H$ such that $x_{0} \notin S$ and $S_{1}+A_{0}+B_{0}+\left[x_{0}+x_{1}\right] \subset$ $S$. Then $x_{0} \in \bar{S}$. Obviously, $x_{1} \notin S$. Let $A=A_{0}^{\perp}$ and $B=B_{0}^{\perp}$ (the complements are taken in $S$ ). Since $A_{0}, B_{0}$ are complete, both $A$ and $B$ belong to $E(S)(=C(S))$. 
We will show that $A \wedge B$ does not exist in $E(S)$. Suppose $A \wedge B$ exists. Then $A \wedge B=A \cap B$. We will prove that $A \cap B$ is neither complete nor cocomplete.

Let us first show that $A \cap B$ is not complete. We start by proving that $\bar{A}=A_{1}$ and $\bar{B}=B_{1}$. Suppose that $\bar{A} \neq A_{1}$ (the case of $\bar{B}$ argues analogous). Since $A \subset \bar{A} \subset A_{1}$ and $\operatorname{dim} A_{1} / A=1$, the strict inclusion $\bar{A} \subset A_{1}$ implies that $A=\bar{A}$. This means that $A$ is complete. Since $A$ is also cocomplete (in $S$ ), then $S=A_{0} \oplus A$ and therefore $S$ is complete. This contradiction shows that $\bar{A}=A_{1}$. Since $S_{1} \subset A_{1} \cap B_{1}$, we obtain $S_{1} \subset A_{1} \cap S=\bar{A} \cap S=A$. Analogously, $S_{1} \subset B$. As a consequence, $S_{1} \subset A \cap B \subset A_{1} \cap B_{1}=S_{1}+\left[x_{1}\right]$. Moreover, $x_{1} \notin S$ and therefore $x_{1} \notin A \cap B$. Hence, $S_{1}=A \cap B$ and $x_{1} \in \bar{S}_{1}$. This means that $x_{1} \in \overline{A \cap B}-(A \cap B)$ and therefore $A \cap B$ is not complete.

Let us show that $A \cap B$ is not cocomplete. Since by Proposition 2.2.6 (ii) we have the equality $\bar{A} \cap \bar{B}=\overline{A \cap B}$, we can consecutively write

$$
(A \cap B)^{\prime}=\overline{A^{\prime}+B^{\prime}}=\overline{\bar{A}^{\prime}+\bar{B}^{\prime}}=\overline{A_{1}^{\prime}+B_{1}^{\prime}}=\overline{A_{0}+B_{0}} .
$$

But $x_{0} \in \overline{A_{0}+B_{0}}$ and $\overline{A_{0}+B_{0}}=(A \cap B)^{\prime}$. Moreover, $(A \cap B)^{\perp}=(A \cap B)^{\prime} \cap S$. Since $A_{0}+B_{0} \subset(A \cap B)^{\prime} \cap S$, we see that $x_{0} \in \overline{A_{0}+B_{0}} \subset \overline{(A \cap B)^{\perp}}$. But $x_{0} \notin S$ and therefore $x_{0} \notin(A \cap B)^{\perp}$. Thus, $(A \cap B)^{\perp}$ is not complete. It follows that $A \cap B$ is not cocomplete. We have proved that $A \cap B$ is neither complete nor cocomplete and therefore $A \cap B$ does not exist in $E(S)$. Thus, $E(S)$ is not a lattice.

We will finally show that there is a dense hyperplane $S$ in $H$ such that $E(S)$ $(=C(S))$ is a lattice.

Theorem 2.2.8. Let $H$ be a separable Hilbert space. Then $H$ possesses a hyperplane, $S$, such that $S$ contains no infinite dimensional complete subspace. As a consequence, $E(S)$ consists of subspaces of $S$ which have finite or cofinite dimension. Thus, $E(S)$ is a modular lattice.

Proof. Since $H$ possesses a countable dense subset and since each complete subset is determined by its (countable) dense subset, we see that there is $2^{\chi_{0}}$ complete infinite dimensional subspace of $H$. Take the initial ordinal, $\omega$, of the cardinality $2^{\chi_{0}}$ and let $\mathcal{C}=\left\{C_{\alpha} \mid C_{\alpha}\right.$ is a complete subspace of $\left.H, \alpha<\omega\right\}$. We will construct a linearly independent family of vectors in $H, F=\left\{v_{\alpha} \mid \alpha<\omega\right\}$, such that for each $\alpha, \alpha<\omega$, we have $v_{\alpha} \in C_{\alpha}$. It is known that the linear dimension of $H$ is $2^{\chi_{0}}$ (see [5]). We can therefore proceed by transfinite induction. For $C_{1}$ we take for $v_{1}$ an arbitrary nonzero vector of $C_{1}$. Suppose that all vectors $v_{\beta}(\beta<\alpha)$ have been constructed. Since card $C_{\alpha}=2^{\chi_{0}}$ and since card $\left\{v_{\beta} \mid \beta<\alpha\right\}>2^{\chi_{0}}$, there must be a vector, $v_{\alpha}$, in $C_{\alpha}$ so that the family $\left\{v_{\beta} \mid \beta \leq \alpha\right\}$ is linearly independent. Having this verified, let us enlarge the entire family $\left\{v_{\beta} \mid \beta<\omega\right\}$ to a linear basis of $H$. Take the linear function, $f$, on $H$ so that $f\left(v_{\beta}\right)=p_{\beta}$ for each $\beta(\beta<\omega)$, where $\left\{p_{\beta} \mid \beta<\omega\right\}$ is an arbitrary unbounded family of positive numbers. Then the set $S=f^{-1}(0)$ is a dense hyperplane of $H$ and, by our construction, $S$ does not contain a complete subspace of infinite dimension.

Let us observe in conclusion that Theorem 2.2.8 allows us to obtain the answer to a question published in 2] (Problem 4.3.13, p. 234).

Let $S$ be an incomplete inner product space and let $\bar{S}$ be its completion (thus, $\bar{S}$ is a Hilbert space). If we consider $E(S)$ as a suborthomodular poset of $E(\bar{S})$ (we can do so since the embedding $e: E(S) \rightarrow E(\bar{S})$ such that $e(A)=\bar{A}$ obviously preserves the complements), a natural question occurs whether there is always a 
state on $E(S)$ which is not a restriction of a "Hilbertian" state on $E(\bar{S})$. Consider the space $S$ constructed in Theorem 2.2.8. We claim that each state on $E(S)$ is a restriction of a state on $E(\bar{S})$, answering the above posed question to the negative. By Theorem 2.2.8 $E(S)$ consists of finite dimensional and cofinite dimensional subspaces of $S$. Since each state on $E(S)$ which lives on a finite dimensional subspace of $S$ obviously is a restriction of a state on $E(S)$, it remains to be shown that so is the (only) "free" state, $s$, on $E(S)$. This state $s$ is determined as follows: $s(A)=1$ if, and only if, $A$ is cofinite dimensional. But there is a state, $\bar{s}$, on $E(\bar{S})$ which vanishes on all finite dimensional subspaces of $\bar{S}$ (see e.g. [10]). It is clear that $s$ is a restriction of $\bar{s}$. This completes the proof.

\section{REFERENCES}

[1] H. Araki Amemiya, A remark on Piron's paper, Publ. Res. Inst. Math. Sci., Kyoto Univ., Ser. A 2 (1966), 423-427. MR 35:4130

[2] A. Dvurečenskij, Gleason's Theorem and Applications, Kluwer Academic Publishers, Dordrecht/Boston/London, 1993. MR 94m:46092

[3] A. Dvurečenskij, S. Pulmannová, States on splitting subspaces and completeness of inner product spaces, Inter. J. Theor. Phys. 27 (1988), 1059-1067. MR 90b:46050

[4] H. Gross, H. Keller, On the definition of Hilbert space, Manuscr. Math. 23 (1977), 67-90. MR 58:2188

[5] P. Halmos, A Hilbert Space Problem Book, Van Nostrand-Reinhold, Princeton, New Jersey, 1967. MR 34:8178

[6] J. Hamhalter, P. Pták, A completeness criterion for inner product spaces, Bull. London Math. Soc. 19 (1987), 259-263. MR 88a:46019

[7] J. Harding, Orthomodular lattices whose MacNeille completions are not orthomodular, Order 8 (1991), 93-103. MR 92k:06015

[8] J. Harding, Decompositions in quantum logic, Trans. Amer. Math. Soc., Vol. 348, 5 (1996), 1839-1862. MR 96h:81003

[9] S. S. Holland, Jr., Orthomodularity in infinite dimensions; a theorem of M. Solér, Bull. Amer. Math. Soc., Vol. 32, No. 2 (1995), 205-234. MR 95j:06010

[10] G. Kalmbach, Measures and Hilbert Lattices, World Sci. Publ. Co., Singapore, 1986. MR 88a:06013

[11] H. A. Keller, Ein nicht-klassischer Hilbertischen Raum, Math. Z. 272 (1980), 42-49. MR 81f:46033

[12] F. Maeda, S. Maeda, Theory of Symmetric Lattices, Springer-Verlag, Berlin, 1970. MR 44:123

[13] R. Mayet, Some characterizations of the underlying division ring of a Hilbert lattice by automorphisms, Int. J. Theor. Phys. 37 (1998), 109-114. MR 99f:81020

[14] R. Piziak, Lattice theory, quadratic spaces, and quantum proposition systems, Found. Phys. 20 (1990), 651-665. MR 91h:06021

[15] P. Pták, S. Pulmannová, Orthomodular Structures as Quantum Logics, Kluwer, 1991. MR 94d:81018b

[16] M. P. Solér, Characterization of Hilbert spaces by orthomodular spaces, Comm. in Algebra 23 (1) (1995), 219-243. MR 95k:46035

[17] V. Varadarajan, Geometry of Quantum Theory I, II, Van Nostrand, Princeton, 1968, 1970. MR 57:11399: MR 57:11400]

Faculty of Electrical Engineering, Department of Mathematics, Czech Technical University, 16627 Prague 6, Czech Republic

E-mail address: ptak@math.feld.cvut.cz

Dipartimento di Matematica, e Informatica, Università degli Studi di Udine, I-33100 Udine, ITALY

E-mail address: weber@dimi.uniud.it 